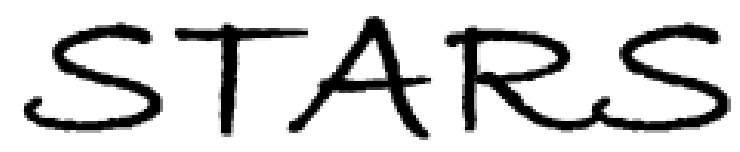

University of Central Florida

STARS

$1-1-2005$

\title{
Low-power, multiport, ultrafast, parametric switching in cascaded waveguide couplers
}

\author{
Roland Schiek \\ Robert Iwanow \\ University of Central Florida \\ George Stegeman \\ University of Central Florida \\ Thomas Pertsch \\ Falk Lederer
}

See next page for additional authors

Find similar works at: https://stars.library.ucf.edu/facultybib2000

University of Central Florida Libraries http://library.ucf.edu

This Article is brought to you for free and open access by the Faculty Bibliography at STARS. It has been accepted for inclusion in Faculty Bibliography 2000s by an authorized administrator of STARS. For more information, please contactSTARS@ucf.edu.

\section{Recommended Citation}

Schiek, Roland; Iwanow, Robert; Stegeman, George; Pertsch, Thomas; Lederer, Falk; Min, Yoo Hong; and Sohler, Wolfgang, "Low-power, multiport, ultrafast, parametric switching in cascaded waveguide couplers" (2005). Faculty Bibliography 2000s. 5639.

https://stars.library.ucf.edu/facultybib2000/5639

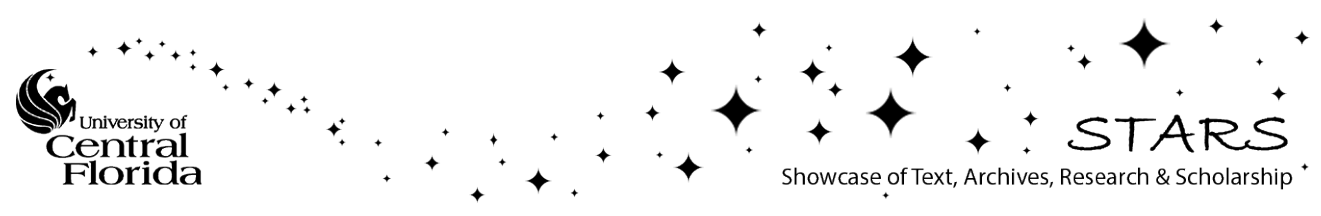




\section{Authors}

Roland Schiek, Robert Iwanow, George Stegeman, Thomas Pertsch, Falk Lederer, Yoo Hong Min, and Wolfgang Sohler 


\title{
Low-power, multiport, ultrafast, parametric switching in cascaded waveguide couplers
}

\author{
Roland Schiek \\ University of Applied Sciences Regensburg, Prüfeninger Str. 58, 93049 Regensburg, Germany \\ Robert Iwanow and George Stegeman \\ Center for Research and Education in Optics and Lasers-School of Optics, University of Central Florida, \\ 4000 Central Florida Boulevard, Orlando, Florida 32816
}

Thomas Pertsch ${ }^{\text {a) }}$ and Falk Lederer

Friedrich-Schiller-Universität Jena, Max-Wien-Platz 1, 07743 Jena, Germany

Yoo Hong Min and Wolfgang Sohler

Applied Physics, Universität Paderborn, 33095 Paderborn, Germany

(Received 11 March 2005; accepted 7 June 2005; published online 30 June 2005)

\begin{abstract}
We explore an approach to multiport, phase-insensitive all-optical switching of milliwatt power signals based on linear coupling in cascaded directional couplers and on nonlinear parametric gain via the second-order nonlinearity. For the basic unit, a two-core coupler, we experimentally demonstrated ultrafast transparent switching of $1550 \mathrm{~nm}$ signals triggered by 5-ps-long control pulses at $\approx 775 \mathrm{~nm}$ with a peak power of $1.3 \mathrm{~W}$. Theoretically, we show that this concept has the potential for terabit/s switching with a few picojoules control energy and without pulse degradation. (C) 2005 American Institute of Physics. [DOI: 10.1063/1.1991993]
\end{abstract}

The nonlinear directional coupler (NLDC), first suggested in the early 1980s, is based on the nonlinear detuning of the coupler arms. ${ }^{1,2}$ To date there is a substantial amount of theoretical literature (for an overview see Ref. 3), aimed at device optimization by altering the geometry as well as the input conditions (e.g., phase controlled switching). Various power controlled switching experiments have been reported using the quasi-instantaneous Kerr nonlinearity in silica fibers, semiconductor waveguides at half the band gap, and polymer waveguides. ${ }^{4-6}$ The concept has been extended towards carrier-induced nonlinearities in semiconductors as well as cascaded quadratic nonlinearities. ${ }^{7-9}$ Regardless of the nonlinear effect exploited, ultrafast (picoseconds and subpicoseconds) power controlled switching in compact NLDCs has serious drawbacks that cannot be overcome and prevent its application in optical networks. These include first, the high required signal power for switching, e.g., $>30 \mathrm{~W}$ in quadratic PPLN periodically poled lithium niobate (PPLN) couplers, ${ }^{9}>100 \mathrm{~W}$ in Kerr media, ${ }^{5}$ etc., and second, the temporal breakup of pulses leading to incomplete switching (about 60\%) and signal degradation. The latter precludes a cascadability of the switch which would be required for multiport operation. Even though temporal solitons could in principle be used to avoid pulse breakup, this approach is so far not feasible except in long fibers and there is no experimental proof of this concept. ${ }^{10}$ Here we suggest a new scheme characterized by low signal power (milliwatt), ultrafast (beyond $100 \mathrm{Gbit} / \mathrm{s}$ ) as well as transparent (lossless) operation, phase insensitivity, no signal degradation, high switching contrast (beyond $30 \mathrm{~dB}$ ), compatibility with wavelength division multiplexed (WDM) systems and cascadability. Finally, we will show that the device obeys simple scaling laws and predict on this basis ultrahigh capacity switching operation with femtosecond pulses.

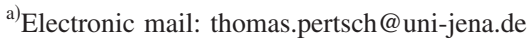

A multiport, PPLN, switching device is shown in Fig. 1. It consists of multiple, cascaded, identical, half-beat-length directional couplers which differ only in the input channels used for the control, shown in Figs. 2(a) and 2(b). A control input at port $C_{n}(n=1,2,3 \ldots)$ switches the low power signal transparently, undistorted and with high discrimination to the respective output port $S_{n}$. Without control input all output ports $S_{n}$ are dark and the signal travels from its input $S$ diagonal through the matrix. Our approach is based on two familiar effects in PPLN waveguides, namely linear signal (wavelength $\lambda_{s}$ ) coupling between two parallel waveguides and parametric gain provided by down conversion of a control pulse $\left(\approx \lambda_{s} / 2\right)$. The combination of both leads to a number of key features. First, the control pulse is strongly confined to the excitation channel and is essentially decoupled from the adjacent guides. The parametric down conversion in the coupler arm with the control input provides directly signal amplitude changes rather than only phase modulations to be converted into amplitude changes by additional phasesensitive elements. The signal power never needs to exceed the milliwatt level, thus the coupling behavior is always linear without nonlinear signal distortions. Second, the nondegenerate mixing process allows phase-insensitive interaction between two input waves (signal and control). Besides the nondegenerate parametric amplification of the signal (transparency) an idler of slightly different wavelength is gener-

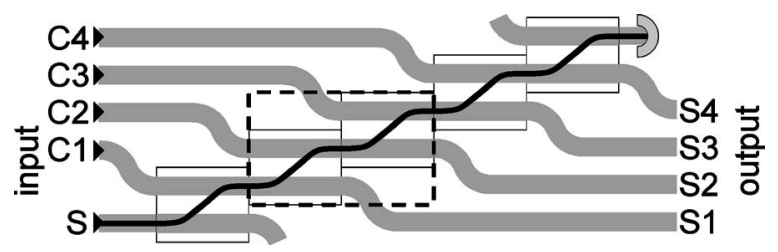

FIG. 1. $1 \times 4$ switching matrix consisting of a sequence of five directional couplers. The basic switching element is marked by the dashed line. $C_{n}$ and $S_{n}$ are the control beam inputs and signal beam outputs, respectively. 

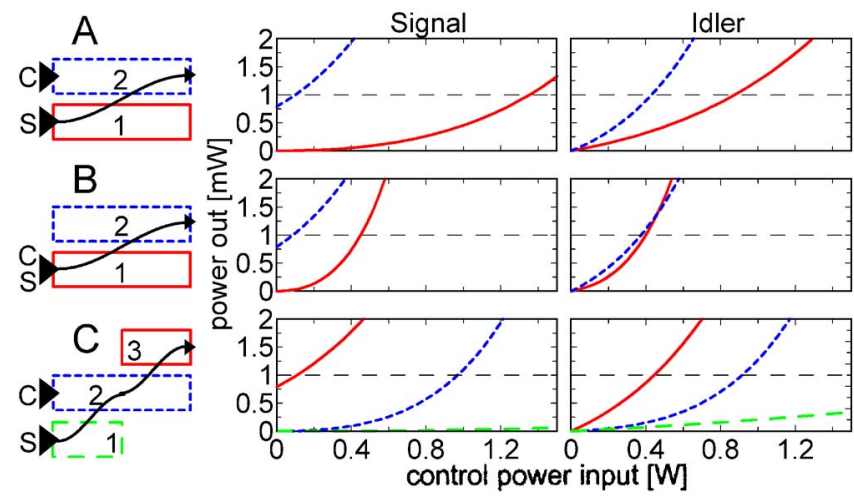

FIG. 2. (Color online) Simulated dependence of the signal and idler output on the control input power for three different coupler configurations (cw excitation). Transparent operation is reached at the dashed line where the signal output power equals the input power. The total device length for all three configurations is $50 \mathrm{~mm}$. In configuration A and B the coupling length is $50 \mathrm{~mm}$, in configuration $\mathrm{C}$ it is $25 \mathrm{~mm}$.

ated with potential for signal-wavelength conversion in a WDM system. Third, a cascade of couplers provides both spatial separation (high contrast), and cascadability. Finally, the power levels required for the control beam are much lower than in conventional NLDCs.

The theoretical modeling of the spatiotemporal response of a basic switching element [indicated by dashed lines in Fig. 1 and shown also in Fig. 2(c)] is based on the coupled mode equations

$$
\begin{aligned}
& i \frac{\partial u_{n}^{C}}{\partial z}-\frac{\beta_{2}^{C}}{2} \frac{\partial^{2} u_{n}^{C}}{\partial t^{2}}+i \delta \frac{\partial u_{n}^{C}}{\partial t}+i \frac{\alpha^{C}}{2} u_{n}^{C}=-\omega^{P} \gamma u_{n}^{S} u_{n}^{I}, \\
& i \frac{\partial u_{n}^{S}}{\partial z}-\frac{\beta_{2}^{S}}{2} \frac{\partial^{2} u_{n}^{S}}{\partial t^{2}}+\kappa u_{3-n}^{S}+i \frac{\alpha^{S}}{2} u_{n}^{S}=-\omega^{S} \gamma u_{n}^{I^{*}} u_{n}^{C}, \\
& i \frac{\partial u_{n}^{I}}{\partial z}-\frac{\beta_{2}^{I}}{2} \frac{\partial^{2} u_{n}^{I}}{\partial t^{2}}+\kappa u_{3-n}^{I}+i \frac{\alpha^{I}}{2} u_{n}^{I}=-\omega^{I} \gamma u_{n}^{S^{*}} u_{n}^{C} .
\end{aligned}
$$

They describe the spatial $(z)$ and temporal $(t)$ evolution of the complex amplitudes $u_{n}^{X}(z, t)$ of the phase-matched fundamental guided modes for the control $(X=C)$, signal $(X=S)$, and idler $(X=I)$ in the two arms of the respective two core coupler $(n=1$ and $n=2)$. $\beta_{2}^{X}$ denotes the group velocity dispersion, $\delta$ is the walkoff between the control and signal (and idler) beams, $\kappa$ is the coupling between parallel guides, $\alpha^{X}$ is the wavelength-dependent loss, $\omega^{X}$ is the frequency, and $\gamma$ is the effective quadratic nonlinearity. The pertinent parameters are given in Ref. 11. In Fig. 2 the dependencies of the signal and idler output on the control input power for different input combinations of control and signal are shown for a cw simulation. In case A, with control and signal input in different coupler arms of a perfect half-beat-length coupler, increasing the control power results in an increasing signal in arm 2 with a much weaker output from arm 1 . Thus this element acts essentially as a parametric preamplifier. Similarly case B, with control and signal input in the same arm of the halfbeat-length coupler, shows a quick rise of the parametrically generated signal and idler in arm 1, i.e., signal and idler appear at an otherwise (no control) dark output. This element provides the switching operation of the combined switching element $(C)$ with a response as displayed in Fig. 2(c). Here, the parametric mixing in the first stage (A) gives rise to

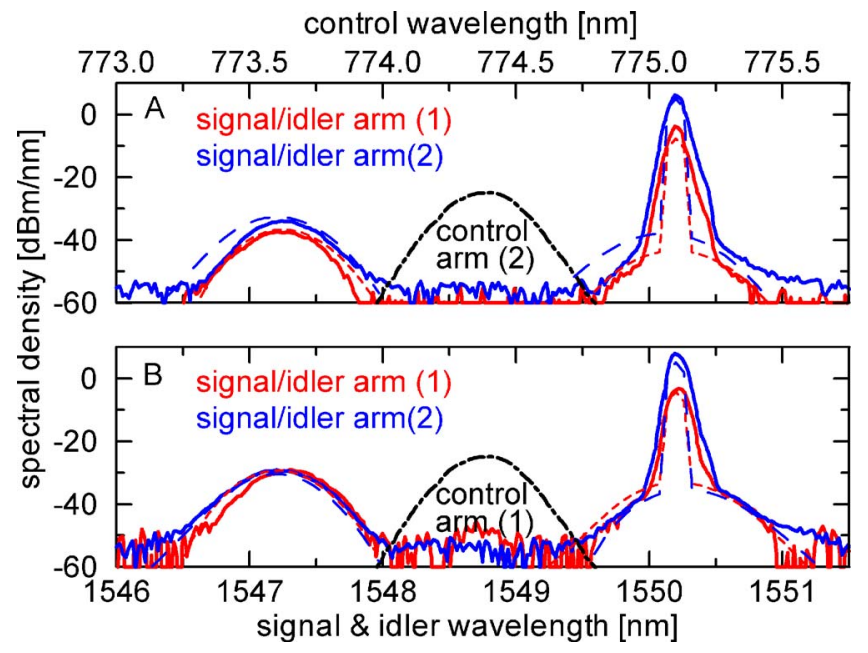

FIG. 3. (Color online) Measured (solid) and simulated (dashed) output spectra of the control, signal, and idler after parametric interaction in the coupler configurations A and B (2 W control input peak power).

signal amplification and idler generation in channel 2 without pronounced crosstalk to an undesirable output from arm 1. In the second stage (B) the primary switching to the output port of interest, arm 2, acts on the already preamplified signal and idler. In a complete switching matrix as in Fig. 1, the "unused" signal and idler in channel 3 will then travel the sequence of subsequent coupler stages without exiting to any other output channel in the absence of additional control signals.

To experimentally verify the idea we fabricated 50 -mm-long couplers by diffusion of $7-\mu \mathrm{m}$-wide and 98 -nm-thick titanium stripes into PPLN with a poling period $16.75 \mu \mathrm{m}$. The coupler with $18.3 \mu \mathrm{m}$ channel separation showed $96 \%$ linear cross coupling at $\lambda=1550 \mathrm{~nm}$ and was the best approximation to a half-beat length coupler for signal and idler on the sample. We used a frequency doubled color center laser as the source of 5-ps-long (full width at half maximum) transform limited Gaussian control pulses $\left(\lambda_{c}=774.38 \mathrm{~nm}\right.$, repetition rate $\left.76 \mathrm{MHz}\right)$. The signal $\left(\lambda_{s}\right.$ $=1550-1560 \mathrm{~nm}$ ) was generated by a separate tunable, $\mathrm{cw}$ diode laser to provide phase-insensitive operation. At the operating temperature of $202{ }^{\circ} \mathrm{C}$ the coupler modes were phase matched for second-harmonic generation of $2 \lambda_{c}$. Using a microscope objective both laser beams were simultaneously focused onto the entrance facet of the PPLN sample in order to excite the $\mathrm{TM}_{00}$ modes in the coupler branches according to Fig. 2. The output was characterized by a spectrum analyzer. Although a cw signal was used to avoid the synchronization of two pulsed laser sources, we did obtain sufficient information about the temporal response of the switch by comparing the spectral characterization data with the numerical modeling.

Measurements were performed with a cw input signal with $0.47 \mathrm{~mW}$ and control pulses with variable peak power between 0.5 and $2 \mathrm{~W}$. Figure 3 shows measured output spectra for control pulses with a peak power of $2 \mathrm{~W}$ for both input configurations A and B for the nearly half-beat-length coupler. The parametrically generated signal and idler pulses are observed as broad spectral lines as compared to the narrow peak of the cw input signal whose observed spectral width is limited by the spectrum analyzer's resolution. Moreover the low duty cycle of the control pulses $\left(3.8 \times 10^{-4}\right)$ 


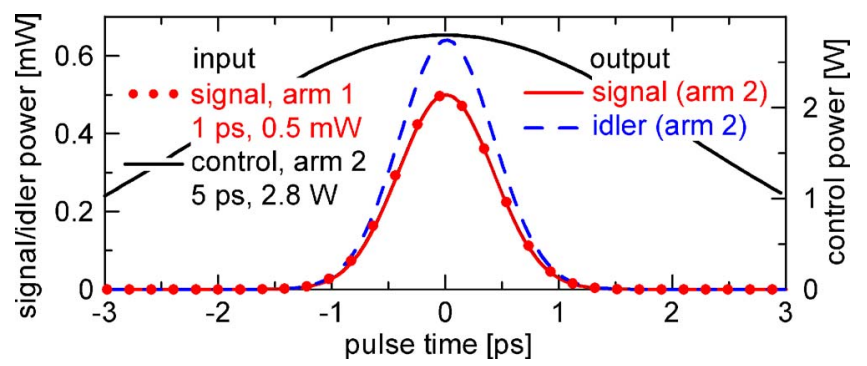

FIG. 4. (Color online) Simulated input and output pulses from configuration C plotted in time frames fixed to the pulse centers.

leads to the much lower spectral density level of the generated pulses as compared to the cw input signal. For both, configurations $\mathrm{A}$ and $\mathrm{B}$, the different amplitudes of the parametrically generated signal and idler in the two arms of the coupler were measured in good agreement with the predictions in Fig. 2, although the power levels in the experiment with pulsed control were increased compared to the cw simulations in Fig. 2 due to the walkoff between amplified and generated signal (and idler) and control. The $\sim 5 \mathrm{~dB}$ difference of the idler in both output channels for input configuration A contrasts the equal idler outputs for configuration B where signal and idler also show higher levels compared to configuration A. In configuration B transparent switching occurs with a control peak power of $1.3 \mathrm{~W}$ when the output signal pulses from arm 1 reach a peak power equal to the $\mathrm{cw}$ input signal power of $0.47 \mathrm{~mW}$. Furthermore, changing the input signal wavelength led to shifting the generated idler wavelength over the parametric gain bandwidth of $20 \mathrm{~nm}$. However, phase matching limits the control acceptance bandwidth to only $0.14 \mathrm{~nm}$. The good quantitative agreement between measurements and time-resolved simulations (shown in Fig. 3 with the dashed lines) for all power level combinations verifies the theoretical model based on Eqs. (1) and illustrates the high quality of the coupler devices fabricated.

Based on the good agreement between experiments (configurations A and B) and numerical simulations, the high bit rate performance for case $\mathrm{C}$ can be predicted by numerical modeling. Figure 4 shows the calculated signal and idler output pulses from arm 2 of configuration $\mathrm{C}$ when 5 ps control and 1 ps signal pulses were launched (signal peak power $0.5 \mathrm{~mW}$ ). For a $50-\mathrm{mm}$-long device, transparent switching without observable pulse degradation was calculated for $2.8 \mathrm{~W}$ control peak power (15 pJ pulse energy). Similar to a short pulse optical parametric amplifier, the broad bandwidth of the parametric gain permits amplification of very short pulses. However, the control acceptance bandwidth, set by the phase-matching condition, and the group-velocity mismatch between the signal (and idler) and the control leading to walkoff are the major limiting factors for this switch. There is a complicated interplay between the switching speed, power, crosstalk, and pulse distortion for a given set of device parameters (length and poling grating). However, it showed in the simulations for Fig. 4 that the spatiotemporal dynamics yielded only minimum pulse degradations. In Fig. 4 the input and output pulses were both transform limited, 1 ps long and equal each other almost perfectly. A proper temporal pulse synchronization permits an optimization of the spatiotemporal overlap of control and signal inside the switching unit leading to a signal crosstalk in arm 1 of only $-43 \mathrm{~dB}$ (for a pulse-to-pulse distance of $10 \mathrm{ps}$ at a data rate of $100 \mathrm{Gbit} / \mathrm{s})$. The switching speed can be increased further by using shorter devices at the expense of increasing the control pulse energy where both, the achievable switching speed and the control pulse energy, scale linear with the device length. Hence, extrapolating to the complete four channel switching matrix in Fig. 1, transparent switching of a $250 \mathrm{Gbit} / \mathrm{s}$ data stream with control pulses of $37 \mathrm{pJ}$ should be feasible in a 50-mm-long PPLN device. Scaling to even shorter basic cells $(C)$, for example $2.5 \mathrm{~mm}$ length, would allow larger switching matrices and transparent switching at terabit/s rates with $150 \mathrm{pJ}$ control pulse energies. Finally, local control of the poling period (phase-matching condition) would permit further engineering of the performance figures.

In conclusion, we have demonstrated phase-insensitive switching in a coupled waveguide configuration. Transparent operation of a single coupler was achieved for $1.3 \mathrm{~W}$ peak power of the control input pulses. Comparing the spectral measurements with the results of detailed simulations we concluded that the switched output pulses suffered almost no pulse distortion. Based on the good correspondence with the experimental results, the theoretical analysis projects that the devices are scalable to switching speeds well beyond $100 \mathrm{Gbit} / \mathrm{s}$. The experimental results suggest that entire switching matrices can be based on this concept.

This research was supported in Europe by the European Commission under Contract No. IST-2000-26005 "ROSA" and in the U.S. by the National Science Foundation and an Army MURI on "Solitonic Gateless Computing."

${ }^{1}$ S. M. Jensen, IEEE J. Quantum Electron. QE-18, 1580 (1982).

${ }^{2}$ A. A. Maier, Sov. J. Quantum Electron. 12, 1490 (1982).

${ }^{3}$ J. S. Aitchison, A. Villeneuve, and G. I. Stegeman, J. Nonlinear Opt. Phys. Mater. 4, 871 (1995).

${ }^{4}$ S. R. Friberg, Y. Silberberg, M. K. Oliver, M. J. Andrejco, M. A. Saifi, and P. W. Smith, Appl. Phys. Lett. 51, 1135 (1987).

${ }^{5}$ G. I. Stegeman and A. Miller, in Photonic Switching, edited by J. Midwinter (Academic, Orlando, 1992), Vol. I, pp. 81-146.

${ }^{6}$ Th. Gabler, A. Bräuer, H.-H. Hörhold, T. Pertsch, and R. Stockmann, Chem. Phys. 245, 507 (1999).

${ }^{7}$ P. Li Kam Wa, J. E. Sitch, N. J. Mason, J. S. Roberts, and P. N. Robson, Electron. Lett. 21, 26 (1885).

${ }^{8}$ R. Jin, J. P. Sokoloff, P. A. Harten, C. L. Chuang, C. G. Lee, M. Warren, H. M. Gibbs, N. Peyghambarian, J. N. Polky, and G. A. Pubanz, Appl. Phys. Lett. 56, 993 (1990).

${ }^{9}$ R. Schiek, L. Friedrich, H. Fang, G. I. Stegeman, K. R. Parameswaran, M.-H. Chou, and M. M. Fejer, Opt. Lett. 24, 1617 (1999).

${ }^{10}$ M. Romagnoli, S. Trillo, and S. Wabnitz, Opt. Quantum Electron. 24, S1237 (1992).

${ }^{11}$ T. Pertsch, R. Iwanow, R. Schiek, G. Stegeman, U. Peschel, F. Lederer, Y. H. Min, and W. Sohler, Opt. Lett. 30, 177 (2005). 